\title{
Interference and oscillation in Nambu quantum mechanics
}

\author{
Djordje Minic $\odot,{ }^{*}$ Tatsu Takeuchi $\odot,^{\dagger}$ and Chia Hsiung Tze ${ }^{\ddagger}$ \\ Center for Neutrino Physics, Department of Physics, Virginia Tech, Blacksburg, Virginia 24061, USA
}

(Received 21 December 2020; accepted 5 August 2021; published 10 September 2021)

\begin{abstract}
Nambu quantum mechanics, proposed in [Phys. Lett. B 536, 305 (2002)], is a deformation of canonical quantum mechanics in which only the time-evolution of the "phases" of energy eigenstates is modified. We discuss the effect this theory will have on oscillation phenomena, and place a bound on the deformation parameters utilizing the data on the atmospheric neutrino mixing angle $\theta_{23}$.
\end{abstract}

DOI: 10.1103/PhysRevD.104.L051301

\section{INTRODUCTION}

Quantum mechanics $(\mathrm{QM})$ is one of the most important and successful frameworks of modern physics. The language of QM is essential for particle, nuclear, atomic, condensed matter, and statistical physics as well as chemistry, and it has lead to the current "second quantum revolution" in quantum information science and technology [1]. Nevertheless, the full understanding of the foundations and origins of QM is still an active area of intense discussion and research [2-4]. It has been argued that canonical QM should be replaced by a more fundamental or generalized framework, either in the context of quantum gravity and cosmology [5,6], or in the realm of quantum measurement [7], or in the domain of macroscopic quantum systems [8].

A deeper understanding of canonical QM could be obtained by comparing its predictions to those of its possible generalizations, and confronting both with experiment. It would allow us to probe the robustness of the original tenet or axiom that was relaxed to generalize the theory, thereby identify the theoretical bedrock on which QM rests.

Various proposals for alternative or generalized QM theories can be found in the literature [9-22]. In this paper, we look at one such generalization, Nambu QM, which was introduced in [23] by Minic and Tze, and one of its observable consequences. The work was inspired by a profound and farreaching paper by Nambu [24], and thus its name.

The starting point of the Nambu QM approach is the geometric formulation of $\mathrm{QM}$ [25] in which the time

\footnotetext{
*dminic@vt.edu

†takeuchi@vt.edu

ctze@me.com
}

Published by the American Physical Society under the terms of the Creative Commons Attribution 4.0 International license. Further distribution of this work must maintain attribution to the author(s) and the published article's title, journal citation, and DOI. Funded by SCOAP ${ }^{3}$. evolution of pure quantum states is described as a "classical" area preserving Hamiltonian flow within the state "phase" space. For a single energy eigenstate, this is just the evolution of its phase, the real and imaginary parts of which constitute the "phase" space, with the Hamiltonian being that of a harmonic oscillator. Nambu's idea in [24] was to extend the classical equation of motion $\dot{F}=-\{H, F\}$, where

$$
\{A, B\}=\varepsilon_{i j} \frac{\partial A}{\partial q_{i}} \frac{\partial B}{\partial q_{j}}
$$

is the Poisson bracket, to $\dot{F}=-\left\{H_{1}, H_{2}, F\right\}$, where

$$
\{A, B, C\}=\varepsilon_{i j k} \frac{\partial A}{\partial q_{i}} \frac{\partial B}{\partial q_{j}} \frac{\partial C}{\partial q_{k}},
$$

i.e., the Nambu bracket. In Poisson dynamics time evolution is generated by the one conserved quantity $H$, while Nambu dynamics requires two: $H_{1}$ and $H_{2}$, and the generated flow is volume preserving. An application of the Nambu equation is the asymmetric top in which the evolution of its angular momentum $\vec{L}$ can be generated by the Nambu bracket with the energy $E$ and total angular momentum $L^{2} / 2$ serving the roles of $H_{1}$ and $H_{2}$. The proposal of [23] was to enlarge the "phase" space of each energy eigenstate from two dimensions to three, and assume that the "classical motion" of the phase was governed by Nambu asymmetric top dynamics instead of that of a Poisson harmonic oscillator. Note that this deformation of canonical QM is particularly attractive since it is minimalistic: it only deforms the time-evolution of the phase of energy eigenstates while everything else is kept fixed. Furthermore, this deformation can be continuously turned on and off by collapsing the three dimensions of the phase space down to the original two.

In the following, we first review canonical QM in the two-component real vector notation and the treatment of 
oscillations in that language. Then, we formulate Nambu $\mathrm{QM}$ as the three-component real vector extension to canonical QM and derive an explicit formula for oscillations in this context. This formula can be probed experimentally, most promisingly in neutrino oscillations.

\section{CANONICAL QM}

Let $|n\rangle$ denote the $n$th eigenstate of the Hamiltonian $\hat{H}$ with eigenvalue $E_{n}=\hbar \omega_{n}$ :

$$
\hat{H}|n\rangle=E_{n}|n\rangle .
$$

A generic state $|\psi(t)\rangle$ can be expanded in terms of $|n\rangle$ as

$$
|\psi(t)\rangle=\sum_{n}|n\rangle \underbrace{\langle n \mid \psi(t)\rangle}_{\psi_{n}(t)}=\sum_{n} \psi_{n}(t)|n\rangle,
$$

where the coefficients $\psi_{n}(t)$ evolve in time as

$$
\psi_{n}(t)=N_{n} e^{-i \omega_{n}\left(t-t_{n}\right)} .
$$

Here, we take $N_{n}$ to be real and positive, and $t_{n}$ is the boundary time at which $\psi_{n}(t)$ is phaseless. The complex valued $\psi_{n}(t)$ can also be expressed as a two-component real vector as

$$
\vec{\psi}_{n}(t) \equiv\left[\begin{array}{c}
\operatorname{Re} \psi_{n}(t) \\
\operatorname{Im} \psi_{n}(t)
\end{array}\right]=N_{n}\left[\begin{array}{c}
\cos \omega_{n}\left(t-t_{n}\right) \\
-\sin \omega_{n}\left(t-t_{n}\right)
\end{array}\right]
$$

The inner product between two states $|\psi\rangle$ and $|\phi\rangle$ in this two-component real vector notation is

$$
\langle\psi \mid \phi\rangle=\sum_{n} \psi_{n}^{*} \phi_{n}=\underbrace{\sum_{n}\left(\vec{\psi}_{n} \cdot \vec{\phi}_{n}\right)}_{g(\psi, \phi)}+\underbrace{i \sum_{n}\left(\vec{\psi}_{n} \times \vec{\phi}_{n}\right)}_{\varepsilon(\psi, \phi)} .
$$

Note that $g(\psi, \phi)$ and $\varepsilon(\psi, \phi)$ depend only on the magnitudes of, and the relative angles between the $\left(\vec{\psi}_{n}, \vec{\phi}_{n}\right)$ pairs. They are invariant under 2D rotations. The absolute value of the inner product squared is then

$$
|\langle\psi \mid \phi\rangle|^{2}=g(\psi, \phi)^{2}+\varepsilon(\psi, \phi)^{2} .
$$

Now, consider two energy eigenstates $|1\rangle$ and $|2\rangle$ and two flavor eigenstates $|\alpha\rangle$ and $|\beta\rangle$ which are related by

$$
\left[\begin{array}{c}
|\alpha\rangle \\
|\beta\rangle
\end{array}\right]=\left[\begin{array}{cc}
c_{\theta} & s_{\theta} \\
-s_{\theta} & c_{\theta}
\end{array}\right]\left[\begin{array}{l}
|1\rangle \\
|2\rangle
\end{array}\right]
$$

where $s_{\theta}=\sin \theta, c_{\theta}=\cos \theta$. In vector notation, we have

$$
\begin{aligned}
& \vec{\alpha}_{1}=c_{\theta} \vec{n}_{0}, \quad \vec{\alpha}_{2}=s_{\theta} \vec{n}_{0}, \\
& \vec{\beta}_{1}=-s_{\theta} \vec{n}_{0}, \quad \vec{\beta}_{2}=c_{\theta} \vec{n}_{0},
\end{aligned}
$$

where $\vec{n}_{0}$ represents a phaseless state:

$$
\vec{n}_{0}=\left[\begin{array}{l}
1 \\
0
\end{array}\right] .
$$

Let $|\psi(0)\rangle=|\alpha\rangle$, that is

$$
\vec{\psi}_{1}(0)=\vec{\alpha}_{1}=c_{\theta} \vec{n}_{0}, \quad \vec{\psi}_{2}(0)=\vec{\alpha}_{2}=s_{\theta} \vec{n}_{0} .
$$

At a later time, these will have evolved into

$$
\vec{\psi}_{1}(t)=c_{\theta} \vec{n}_{1}(t), \quad \vec{\psi}_{2}(t)=s_{\theta} \vec{n}_{2}(t),
$$

where

$$
\vec{n}_{1}(t)=\left[\begin{array}{c}
c_{1} \\
-s_{1}
\end{array}\right], \quad \vec{n}_{2}(t)=\left[\begin{array}{c}
c_{2} \\
-s_{2}
\end{array}\right],
$$

with $s_{i}=\sin \omega_{i} t$ and $c_{i}=\cos \omega_{i} t$. To find the survival probability $P(\alpha \rightarrow \alpha)$ of flavor $\alpha$, and the transition probability $P(\alpha \rightarrow \beta)$ to flavor $\beta$, we need $\langle\alpha \mid \psi(t)\rangle$ and $\langle\beta \mid \psi(t)\rangle$. The symmetric and antisymmetric parts of these inner products are

$g(\alpha, \psi(t))=\vec{\alpha}_{1} \cdot \vec{\psi}_{1}(t)+\vec{\alpha}_{2} \cdot \vec{\psi}_{2}(t)=c_{\theta}^{2} c_{1}+s_{\theta}^{2} c_{2}$,

$\varepsilon(\alpha, \psi(t))=\vec{\alpha}_{1} \times \vec{\psi}_{1}(t)+\vec{\alpha}_{2} \times \vec{\psi}_{2}(t)=-c_{\theta}^{2} s_{1}-s_{\theta}^{2} s_{2}$,

$g(\beta, \psi(t))=\vec{\beta}_{1} \cdot \vec{\psi}_{1}(t)+\vec{\beta}_{2} \cdot \vec{\psi}_{2}(t)=-s_{\theta} c_{\theta} c_{1}+s_{\theta} c_{\theta} c_{2}$,

$\varepsilon(\beta, \psi(t))=\vec{\beta}_{1} \times \vec{\psi}_{1}(t)+\vec{\beta}_{2} \times \vec{\psi}_{2}(t)=s_{\theta} c_{\theta} s_{1}-s_{\theta} c_{\theta} s_{2}$,

and the survival and transition probabilities will be

$$
\begin{aligned}
P(\alpha \rightarrow \alpha) & =|\langle\alpha \mid \psi(t)\rangle|^{2}=g(\alpha, \psi(t))^{2}+\varepsilon(\alpha, \psi(t))^{2} \\
& =1-P(\alpha \rightarrow \beta), \\
P(\alpha \rightarrow \beta) & =|\langle\beta \mid \psi(t)\rangle|^{2}=g(\beta, \psi(t))^{2}+\varepsilon(\beta, \psi(t))^{2} \\
& =\sin ^{2} 2 \theta \sin ^{2}\left[\frac{\left(\omega_{1}-\omega_{2}\right) t}{2}\right] .
\end{aligned}
$$

Making the relativistic replacement

$$
\omega_{i} t \rightarrow\left(\omega_{i} t-k_{i} L\right) \stackrel{\text { natural units }}{\longrightarrow}\left(E_{i} t-p_{i} L\right),
$$

and assuming that the energies of the two states are common, $E_{1}=E_{2}=E \gg m_{i}$, we have

$$
\left(E t-p_{i} L\right) \approx E(t-L)+\frac{m_{i}^{2}}{2 E} L,
$$

leading to the identification

$$
\left(\omega_{1}-\omega_{2}\right) t \rightarrow \frac{\delta m_{12}^{2}}{2 E} L \equiv \Delta_{12} .
$$


This gives us the familiar neutrino oscillation formula

$$
P(\alpha \rightarrow \beta)=\sin ^{2} 2 \theta \sin ^{2} \frac{\Delta_{12}}{2} .
$$

\section{NAMBU QM}

The deformation of canonical QM which is detailed in Ref. [23], i.e., Nambu QM, can be summarized as follows. Extend the two-component real vector $\vec{\psi}_{n}$ introduced above to a three-component real vector $\vec{\Psi}_{n}$ :

$$
\vec{\psi}_{n} \rightarrow \vec{\Psi}_{n}
$$

In the two-component case, the components evolved as Eq. (6). For the three-component extension, it is assumed that

$$
\vec{\Psi}_{n}(t)=N_{n}\left[\begin{array}{c}
c_{\xi} \operatorname{cn}\left(\Omega_{n}\left(t-t_{n}\right), k\right) \\
-\kappa c_{\xi} \operatorname{sn}\left(\Omega_{n}\left(t-t_{n}\right), k\right) \\
-s_{\xi} \operatorname{dn}\left(\Omega_{n}\left(t-t_{n}\right), k\right)
\end{array}\right],
$$

where $\mathrm{cn}(u, k), \operatorname{sn}(u, k)$, and $\operatorname{dn}(u, k)$ are Jacobi's elliptical functions [26], and $s_{\xi}=\sin \xi, c_{\xi}=\cos \xi$, and $\kappa=\sqrt{1+k^{2} \tan ^{2} \xi}$. The period of $\operatorname{cn}(u, k)$ and $\operatorname{sn}(u, k)$ in $u$ is $4 K$, where $K=K(k)$ is the complete elliptical integral of the first kind [27], and $\Omega_{n}=(2 K / \pi) \omega_{n}$. The two parameters $k$ and $\xi$ are the deformation parameters, and when they are both set to zero, the time evolution of the first two components of $\vec{\Psi}_{n}$ reduce to that of the two components of $\vec{\psi}_{n}$, while the third component of $\vec{\Psi}_{n}$ vanishes. In principle, we can make the deformation parameters $k$ and $\xi$ depend on $n$, but for the sake of simplicity, we keep them common to all $n$.

Note that the time evolution assumed in Eq. (22) is that of the angular momentum vector $\vec{L}$ of a free asymmetric top in its corotating frame [28]. Though the equations that govern this motion are nonlinear (or more precisely multilinear), the presence of the two conserved quantities of energy $E$ and angular momentum $L^{2}$ renders the motion solvable, norm-preserving, and periodic. $\vec{L}$ evolves along the intersection of the sphere $L^{2}=$ constant and the ellipsoid $E=$ constant. Due to the norm preserving nature of Eq. (22), this time evolution is unitary. However, the time evolution operator cannot be expressed as a matrix as in canonical QM (except when $k=0$ ) due to the evolution being non-linear. In essence, the "phase" of the state evolves periodically on $S^{2}$ instead of on $S^{1}$.

The symmetric and antisymmetric parts of the inner product between two states are extended to

$$
\begin{aligned}
& g(\Psi, \Phi)=\sum_{n}\left(\vec{\Psi}_{n} \cdot \vec{\Phi}_{n}\right) \\
& \vec{\varepsilon}(\Psi, \Phi)=\sum_{n}\left(\vec{\Psi}_{n} \times \vec{\Phi}_{n}\right)
\end{aligned}
$$

where the dot and cross products are now defined in three dimensions. The square of the absolute value of $\langle\Psi \mid \Phi\rangle$ is extended to

$$
|\langle\Psi \mid \Phi\rangle|^{2}=g(\Psi, \Phi)^{2}+\vec{\varepsilon}(\Psi, \Phi) \cdot \vec{\varepsilon}(\Psi, \Phi),
$$

which is invariant under 3D rotations of the phase space.

As demonstrated in [23], this definition of the inner product is equivalent to that in quaternionic QM [10] in which the coefficients are restricted to purely imaginary quaternions, the three parts of which are given by the three components of the phase vector, and

$$
\begin{aligned}
\langle\Psi \mid \Phi\rangle= & g(\Psi, \Phi)-i \varepsilon_{1}(\Psi, \Phi)-j \varepsilon_{2}(\Psi, \Phi) \\
& -k \varepsilon_{3}(\Psi, \Phi) .
\end{aligned}
$$

This correspondence guarantees the mathematical consistency and unitarity of the resulting theory.

Equation (24) allows us to make predictions based on Nambu QM. Since the deformation is in the time-evolution of the phase of each energy eigenstate, we can expect deviations from canonical QM to occur in phenomena that involve the evolution of interference terms.

Consequently, let us look at oscillation in Nambu QM. We consider flavor eigenstates $|\alpha\rangle$ and $|\beta\rangle$ to be superpositions of energy eigenstates $|1\rangle$ and $|2\rangle$ as in Eq. (9). The three-component vector notation of $|\alpha\rangle$ and $|\beta\rangle$ are formally the same as Eq. (10), except with $\vec{n}_{0}$ replaced by the three component object

$$
\vec{n}_{0}=\left[\begin{array}{c}
c_{\xi} \\
0 \\
-s_{\xi}
\end{array}\right]
$$

This corresponds to a "zero phase" state. To clarify that we are working in the three-component formalism, we will replace the label $\alpha$ with $A$, and $\beta$ with $B$ in the following.

Let $|\Psi(0)\rangle=|A\rangle$, that is

$$
\vec{\Psi}_{1}(0)=c_{\theta} \vec{n}_{0}, \quad \vec{\Psi}_{2}(0)=s_{\theta} \vec{n}_{0}
$$

At a later time $t$, these will evolve to

$$
\vec{\Psi}_{1}(t)=c_{\theta} \vec{n}_{1}(t), \quad \vec{\Psi}_{2}(t)=s_{\theta} \vec{n}_{2}(t),
$$

where 


$$
\vec{n}_{i}(t)=\left[\begin{array}{c}
c_{\xi} \mathrm{cn}_{i} \\
-\kappa c_{\xi} \mathrm{sn}_{i} \\
-s_{\xi} \mathrm{dn}_{i}
\end{array}\right],
$$

with $\mathrm{sn}_{i}=\mathrm{sn}\left(\Omega_{i} t, k\right), \mathrm{cn}_{i}=\mathrm{cn}\left(\Omega_{i} t, k\right), \mathrm{dn}_{i}=\mathrm{dn}\left(\Omega_{i} t, k\right)$. The symmetric parts of $\langle A \mid \Psi(t)\rangle$ and $\langle B \mid \Psi(t)\rangle$ are

$$
\begin{aligned}
g(A, \Psi(t))= & \vec{A}_{1} \cdot \vec{\Psi}_{1}(t)+\vec{A}_{2} \cdot \vec{\Psi}_{2}(t) \\
= & c_{\theta}^{2}\left(c_{\xi}^{2} \mathrm{cn}_{1}+s_{\xi}^{2} \operatorname{dn}_{1}\right)+s_{\theta}^{2}\left(c_{\xi}^{2} \mathrm{cn}_{2}+s_{\xi}^{2} \operatorname{dn}_{2}\right), \\
g(B, \Psi(t))= & \vec{B}_{1} \cdot \vec{\Psi}_{1}(t)+\vec{B}_{2} \cdot \vec{\Psi}_{2}(t) \\
= & -s_{\theta} c_{\theta}\left(c_{\xi}^{2} \mathrm{cn}_{1}+s_{\xi}^{2} \mathrm{dn}_{1}\right) \\
& +s_{\theta} c_{\theta}\left(c_{\xi}^{2} \mathrm{cn}_{2}+s_{\xi}^{2} \operatorname{dn}_{2}\right),
\end{aligned}
$$

while the antisymmetric parts are

$$
\begin{aligned}
\vec{\varepsilon}(A, \Psi(t)) & =\vec{A}_{1} \times \vec{\Psi}_{1}(t)+\vec{A}_{2} \times \vec{\Psi}_{2}(t) \\
& =c_{\theta}^{2}\left[\begin{array}{c}
-\kappa s_{\xi} c_{\xi} \mathrm{sn}_{1} \\
s_{\xi} c_{\xi}\left(\mathrm{dn}_{1}-\mathrm{cn}_{1}\right) \\
-\kappa c_{\xi}^{2} \mathrm{sn}_{1}
\end{array}\right]+s_{\theta}^{2}\left[\begin{array}{c}
-\kappa s_{\xi} c_{\xi} \mathrm{sn}_{2} \\
s_{\xi} c_{\xi}\left(\mathrm{dn}_{2}-\mathrm{cn}_{2}\right) \\
-\kappa c_{\xi}^{2} \mathrm{sn}_{2}
\end{array}\right],
\end{aligned}
$$

$\vec{\varepsilon}(B, \Psi(t))=\vec{B}_{1} \times \vec{\Psi}_{1}(t)+\vec{B}_{2} \times \vec{\Psi}_{2}(t)$

$$
\begin{gathered}
=-s_{\theta} c_{\theta}\left[\begin{array}{c}
-\kappa s_{\xi} c_{\xi} \mathrm{sn}_{1} \\
s_{\xi} c_{\xi}\left(\mathrm{dn}_{1}-\mathrm{cn}_{1}\right) \\
-\kappa c_{\xi}^{2} \mathrm{sn}_{1}
\end{array}\right] \\
+s_{\theta} c_{\theta}\left[\begin{array}{c}
-\kappa s_{\xi} c_{\xi} \mathrm{sn}_{2} \\
s_{\xi} c_{\xi}\left(\mathrm{dn}_{2}-\mathrm{cn}_{2}\right) \\
-\kappa c_{\xi}^{2} \mathrm{sn}_{2}
\end{array}\right] .
\end{gathered}
$$

From these expressions, we find the survival and transition probabilities to be

$$
\begin{aligned}
P(A \rightarrow A) & =g(A, \Psi(t))^{2}+\vec{\varepsilon}(A, \Psi(t)) \cdot \vec{\varepsilon}(A, \Psi(t))=1-P(A \rightarrow B) \\
P(A \rightarrow B) & =g(B, \Psi(t))^{2}+\vec{\varepsilon}(B, \Psi(t)) \cdot \vec{\varepsilon}(B, \Psi(t)) \\
& =\sin ^{2} 2 \theta\left[\frac{1-\left\{c_{\xi}^{2}\left(\mathrm{sn}_{1} \mathrm{sn}_{2}+\mathrm{cn}_{1} \mathrm{cn}_{2}\right)+s_{\xi}^{2}\left(k^{2} \mathrm{sn}_{1} \mathrm{sn}_{2}+\mathrm{dn}_{1} \mathrm{dn}_{2}\right)\right\}}{2}\right]
\end{aligned}
$$

For the ease of comparison with Eq. (20), we expand the Jacobi functions in powers of $k^{2}$ [29-31]:

$$
\begin{aligned}
& \operatorname{sn}(\Omega t, k)=\left(1+\frac{k^{2}}{16}\right) \sin (\omega t)+\frac{k^{2}}{16} \sin (3 \omega t)+\cdots, \\
& \operatorname{cn}(\Omega t, k)=\left(1-\frac{k^{2}}{16}\right) \cos (\omega t)+\frac{k^{2}}{16} \cos (3 \omega t)+\cdots, \\
& \operatorname{dn}(\Omega t, k)=\left(1-\frac{k^{2}}{4}\right)+\frac{k^{2}}{4} \cos (2 \omega t)+\cdots,
\end{aligned}
$$

from which we find to order $k^{2}$

$$
\begin{aligned}
\mathrm{sn}_{1} \mathrm{sn}_{2}+\mathrm{cn}_{1} \mathrm{cn}_{2} & =\cos \Delta_{12}-\frac{k^{2}}{4} \cos \Sigma_{12} \sin ^{2} \Delta_{12}, \\
k^{2} \mathrm{sn}_{1} \mathrm{sn}_{2}+\mathrm{dn}_{1} \mathrm{dn}_{2} & =1-k^{2}\left(1+\cos \Sigma_{12}\right) \sin ^{2} \frac{\Delta_{12}}{2},
\end{aligned}
$$

where $\Delta_{12}=\left(\omega_{1}-\omega_{2}\right) t$ and $\Sigma_{12}=\left(\omega_{1}+\omega_{2}\right) t$. Averaging over time makes the $\cos \Sigma_{12}$ terms vanish. Therefore,

$$
P(A \rightarrow B)=\left(c_{\xi}^{2}+s_{\xi}^{2} \frac{k^{2}}{2}\right) \sin ^{2} 2 \theta \sin ^{2} \frac{\Delta_{12}}{2} .
$$

Note that $0 \leq k^{2}<1$. Thus, the effect of the Nambu deformation is an overall suppression factor compared to the undeformed canonical case, Eq. (20). This is the main result of this paper.

\section{DISCUSSION}

In this paper, we consider Nambu QM, a deformation of canonical QM in which the phase space of energy eigenstates is enlarged from $2 \mathrm{D}$ to $3 \mathrm{D}$, and the phase dynamics is deformed from that of a harmonic oscillator to that of an asymmetric top. This deformation maintains the Born rule, i.e., the conservation of norm, which is embedded in the classical dynamics of the phase. The invariance of physical predictions on 2D rotations of the phase space is modified to that under $3 \mathrm{D}$ rotations, a feature responsible for the projectivity of the state space. (Note that we cannot associate a phase shift with a constant shift of $\Omega t$ in Eq. (22).) This invariance can, in principle, be gauged in the field theoretic version of Nambu QM, but since the symmetry is $S O(3)$, it could lead to non-Abelian features though only the phase of a single field will be gauged. The $S^{2}$ geometry of the phase space, as opposed to the canonical $S^{1}$, also suggests that the path integral of Nambu QM is not the usual integral of $e^{i S}$.

We investigate the effect of the Nambu QM deformation on oscillation phenomena and obtain Eq. (35), which can be compared directly to neutrino oscillation. Application to meson oscillations would require further considerations of meson decay and $C P$ violation $[32,33]$. Given that the 
$\sin ^{2} 2 \theta$ term cannot increase beyond one, the suppression factor cannot always be absorbed into $\theta$. For instance, the current bound on the atmospheric mixing angle gives $\sin ^{2} 2 \theta_{23}>0.973,0.963,0.952$ respectively at 1,2 , and $3 \sigma$ for normal ordering [34]. This indicates

$$
s_{\xi}^{2}\left(1-\frac{k^{2}}{2}\right)<0.027(1 \sigma), \quad 0.037(2 \sigma), \quad 0.048(3 \sigma),
$$

though the value of $\theta_{23}$ itself is not yet precisely known. Future improvements in the determination of $\theta_{23}$ at IceCube [35], JUNO [36], and DUNE [37] could improve upon this bound.

Note that oscillation is but one possible phenomenon that could be affected by Nambu QM. There may be many others involving interference and the resulting correlations given that the phase vectors are assumed to move in a very particular way on $S^{2}$. For instance, Refs. [38,39] consider an experiment which probes the difference between canonical and quaternionic QM, which may also be sensitive to the Nambu QM deformation. Such experiments could shed new light on various issues in quantum foundations and in entanglement, and call for a thorough investigation.

Apart from these phenomenological considerations, we would like to highlight the fact that the original paper of Nambu [24] has inspired very many works on the mathematical and foundational nature of the Nambu bracket, Eq. (2), and its related structures [40-42], and on the quantization of those structures and their relevance in string theory (see [43-54] and references therein). More recently, such a structure was discovered [55-58] in the context of a new formulation of nonperturbative string theory and quantum gravity based on quantum spacetime [59-66].

We also note that analogies with the asymmetric top are ubiquitous in various classical and quantum physical systems [28]. This has particularly been the case in phenomenological particle physics. What we have uncovered here in Nambu QM relates closely to, and formally extends in a new direction the top like Hamiltonians used in dynamical models of neutrino oscillations [67,68]. They belong to the family of integrable quantum spin Gaudin models of wider applications in condensed matter physics. The time oscillatory features we have deduced in this letter along with the above mentioned connection further suggests the construction and phenomenological testing of a family of dynamical, integrable $S O(3)$ Nambu top models of, say, neutrinos oscillations with, not a trigonometric but a novel Jacobian elliptic time evolutions with two periodspresumably with one period being much, much smaller than the other. They would add a new prediction for neutrino oscillations in our quest to see theoretically and experimentally beyond the Standard Model.

\section{ACKNOWLEDGMENTS}

We thank P. Huber and R. Pestes for helpful discussions. D. M. and T. T. are supported in part by the DOE (Grant No. DE-SC0020262). D. M. is also supported by the Julian Schwinger Foundation, and T. T. by the NSF (Grant No. PHY-1413031).
[1] I. H. Deutsch, PRX Quantum 1, 020101 (2020).

[2] J. Bell, Speakable and Unspeakable in Quantum Mechanics (Cambridge University Press, Cambridge, England, 2004).

[3] Y. Aharonov and D. Rohrlich, Quantum Paradoxes: Quantum Theory for the Perplexed (Wiley-VCH, New York, 2005).

[4] G. 't Hooft, arXiv:1405.1548.

[5] R. Penrose, Found. Phys. 44, 557 (2014).

[6] M. Gell-Mann and J. B. Hartle, Phys. Rev. A 89, 052125 (2014).

[7] S. Weinberg, Phys. Rev. A 93, 032124 (2016).

[8] A. Leggett, Prog. Theor. Phys. Suppl. 170, 100 (2007).

[9] E. C. G. Stueckelberg, Helv. Phys. Acta 33, 727 (1960), https://www.e-periodica.ch/cntmng?pid=hpa-001\%3A1960 $\% 3 \mathrm{~A} 33 \% 3 \mathrm{~A} \% 3 \mathrm{~A} 1097$.

[10] S. L. Adler, Quaternionic Quantum Mechanics and Quantum Fields (Oxford University Press, Oxford, UK, 1995).

[11] M. Gunaydin, C. Piron, and H. Ruegg, Commun. Math. Phys. 61, 69 (1978).

[12] S. Okubo, Introduction to Octonion and Other NonAssociative Algebras in Physics, Montroll Memorial
Lecture Series in Mathematical Physics (Cambridge University Press, Cambridge, England, 2011).

[13] F. Gursey and C. H. Tze, On the Role of Division, Jordan and Related Algebras in Particle Physics (World Scientific, Singapore, 1996).

[14] L. N. Chang, Z. Lewis, D. Minic, and T. Takeuchi, Mod. Phys. Lett. B 27, 1350064 (2013).

[15] L. N. Chang, Z. Lewis, D. Minic, and T. Takeuchi, J. Phys. A 46, 065304 (2013).

[16] L. N. Chang, Z. Lewis, D. Minic, and T. Takeuchi, J. Phys. A 46, 485306 (2013).

[17] T. Takeuchi, L. N. Chang, Z. Lewis, and D. Minic, AIP Conf. Proc. 1508, 502 (2012).

[18] L. N. Chang, Z. Lewis, D. Minic, and T. Takeuchi, Int. J. Mod. Phys. A 29, 1430006 (2014).

[19] L. N. Chang, Z. Lewis, D. Minic, and T. Takeuchi, J. Phys. A 47, 405304 (2014).

[20] L. N. Chang, D. Minic, and T. Takeuchi, J. Phys. Conf. Ser. 1275, 012036 (2019).

[21] S. Weinberg, Phys. Rev. Lett. 62, 485 (1989).

[22] S. Weinberg, Ann. Phys. (N.Y.) 194, 336 (1989). 
[23] D. Minic and C. H. Tze, Phys. Lett. B 536, 305 (2002).

[24] Y. Nambu, Phys. Rev. D 7, 2405 (1973).

[25] T. Kibble, Commun. Math. Phys. 65, 189 (1979).

[26] Mathematica encodes $\operatorname{sn}(u, k), \operatorname{cn}(u, k)$, and $\operatorname{dn}(u, k)$ respectively as JacobiSN $[u, m]$, JacobiCN $[u, m]$, and JacobiDN $[u, m]$ with $m=k^{2}$.

[27] Mathematica encodes $K(k)$ as EllipticK $[m]$ with $m=k^{2}$.

[28] T. Opatrný, L. Richterek, and M. Opatrný, Sci. Rep. 8, 1984 (2018).

[29] I. D. Gradshteyn and I. M. Ryzhik, Table of Integrals, Series, and Products, corrected and enlarged ed., edited by A. Jeffery (Academic Press, New York, 2014).

[30] M. Abramowitz and I. Stegun, Handbook of Mathematical Functions with Formulas, Graphs, and Mathematical Tables (Martino publishing, Mansfield Centre, CT, 2014).

[31] E. T. Whittaker and G. N. Watson, A Course of Modern Analysis, 3rd ed. (Dover Publications, New York, 2020).

[32] U. Nierste, in Proceedings of the Helmholz International School "Heavy Quark Physics" (HPQ08), edited by A. Ali and M. Ivanov (DESY, Hamburg, Germany, 2009), pp. 1-37 [arXiv:0904.1869].

[33] B. Kayser, AIP Conf. Proc. 1441, 464 (2012).

[34] P. de Salas, D. Forero, S. Gariazzo, P. Martínez-Miravé, O. Mena, C. Ternes, M. Tórtola, and J. Valle, J. High Energy Phys. 02 (2021) 071.

[35] A. Terliuk (IceCube Collaboration), Proc. Sci., NOW2018 (2019) 007.

[36] W.-1. Guo (JUNO Collaboration), J. Phys. Conf. Ser. 888, 012205 (2017).

[37] A. Higuera (DUNE Collaboration), Proc. Sci., EPSHEP2017 (2018) 115.

[38] A. Peres, Phys. Rev. Lett. 42, 683 (1979).

[39] S. Gstir, E. Chan, T. Eichelkraut, A. Szameit, R. Keil, and G. Weihs, arXiv:2104.11577.

[40] L. Takhtajan, Commun. Math. Phys. 160, 295 (1994).

[41] G. Dito, M. Flato, D. Sternheimer, and L. Takhtajan, Commun. Math. Phys. 183, 1 (1997).

[42] G. Dito and M. Flato, Lett. Math. Phys. 39, 107 (1997).

[43] E. Bergshoeff, E. Sezgin, Y. Tanii, and P. Townsend, Ann. Phys. (N.Y.) 199, 340 (1990).

[44] H. Awata and D. Minic, J. High Energy Phys. 04 (1998) 006.

[45] H. Awata, M. Li, D. Minic, and T. Yoneya, J. High Energy Phys. 02 (2001) 013.
[46] K. Fujikawa and K. Okuyama, Phys. Lett. B 411, 261 (1997).

[47] L. Smolin, Phys. Rev. D 57, 6216 (1998).

[48] T. L. Curtright and C. K. Zachos, Phys. Rev. D 68, 085001 (2003).

[49] T. L. Curtright and C. K. Zachos, New J. Phys. 4, 83 (2002).

[50] T. L. Curtright and C. K. Zachos, AIP Conf. Proc. 672, 165 (2003).

[51] J. A. Bagger and N. Lambert, Phys. Rev. D 77, 065008 (2008).

[52] A. Gustavsson, Nucl. Phys. B811, 66 (2009).

[53] C.-S. Chu, P.-M. Ho, Y. Matsuo, and S. Shiba, J. High Energy Phys. 08 (2008) 076.

[54] P.-M. Ho and Y. Matsuo, Prog. Theor. Exp. Phys. 2016, 06A104 (2016).

[55] L. Freidel, R. G. Leigh, and D. Minic, Int. J. Mod. Phys. A 34, 1941004 (2019).

[56] D. Minic, in 10th Mathematical Physics Meeting: School and Conference on Modern Mathematical Physics (2020), pp. 183-218 [arXiv:2003.00318].

[57] P. Berglund, T. Hübsch, and D. Minić, Phys. Lett. B 798, 134950 (2019).

[58] P. Berglund, T. Hübsch, and D. Minic, Lett. High Energy Phys. 2021, 186 (2021).

[59] L. Freidel, R. G. Leigh, and D. Minic, Phys. Lett. B 730, 302 (2014).

[60] L. Freidel, R. G. Leigh, and D. Minic, Int. J. Mod. Phys. D 23, 1442006 (2014).

[61] L. Freidel, R. G. Leigh, and D. Minic, J. High Energy Phys. 06 (2015) 006.

[62] L. Freidel, R. G. Leigh, and D. Minic, Phys. Rev. D 94, 104052 (2016).

[63] L. Freidel, R. G. Leigh, and D. Minic, J. Phys. Conf. Ser. 804, 012032 (2017).

[64] L. Freidel, R. G. Leigh, and D. Minic, J. High Energy Phys. 09 (2017) 060.

[65] L. Freidel, R. G. Leigh, and D. Minic, Phys. Rev. D 96, 066003 (2017).

[66] L. Freidel, J. Kowalski-Glikman, R. G. Leigh, and D. Minic, Phys. Rev. D 99, 066011 (2019).

[67] G. G. Raffelt, Phys. Rev. D 83, 105022 (2011).

[68] Y. Pehlivan, A. B. Balantekin, T. Kajino, and T. Yoshida, Phys. Rev. D 84, 065008 (2011). 